\title{
Patient selection for an upper extremity neuroprosthesis in tetraplegic individuals
}

\author{
Peter H Gorman ${ }^{1}$, Kathy Stroh Wuolle ${ }^{2}$, P Hunter Peckham ${ }^{2}$ and David Heydrick ${ }^{1}$ \\ ${ }^{1}$ Physical Medicine and Rehabilitation Service, VA Maryland Health Care System and University of Maryland \\ Medical System, Baltimore, Maryland; ${ }^{2}$ MetroHealth Medical Center and Cleveland VA Medical Center, Case \\ Western Reserve University, Cleveland, Ohio, USA
}

\begin{abstract}
One hundred and twenty persons with new onset traumatic tetraplegia consecutively admitted to our rehabilitation service were screened for consideration for use of an upper extremity neuroprosthesis. Strict inclusion criteria allowed only for participation of patients with ASIA impairment scale A, B or C injuries at the C5 or C6 level. One hundred and six persons were excluded from participation for the following reasons: five patients died, 27 had central cord syndrome, two had Brown-Sequard syndrome, 12 were injured at too high a level, 42 were injured at too low a level, two were excluded on the basis of motor incompleteness alone, four were excessively denervated, two had limited range of motion, one had overriding medical complications, seven had psychosocial issues making participation impractical, and two elected tendon transfer surgery. In total, 14 patients (representing $11.7 \%$ of all tetraplegic individuals and $50 \%$ of the C5 or C6 ASIA Impairment Scale A, B or C patients) were found to be candidates for the neuroprosthesis. Given the prevalence of tetraplegia, approximately 12,200 Americans would be candidates for the FES neuroprosthetic hand grasp system under the current research protocols. With both the expansion of current protocols to other diagnostic categories and further research and development, application of this neuroprosthesis to a considerable number of previously excluded subjects will likely be possible.
\end{abstract}

Keywords: tetraplegia; functional electrical stimulation; neuroprosthesis; hand grasp; eligibility criteria

\section{Introduction}

The individual with tetraplegia experiences many challenges in an effort to return to a productive and satisfying life after spinal cord injury (SCI). Through surveys of this group of patients, studies have shown that one of the most difficult aspects of life after SCI is compensation for the loss of hand function. One study reported that a majority of tetraplegic persons consider this to be the most devastating aspect of their disability. ${ }^{1}$ It is therefore logical that a great degree of effort in the medical rehabilitation community has occurred to help restore some aspects of hand function to this patient group. One of the major thrusts of research in this area has been the application of the technology of functional electrical stimulation (FES).

The use of FES for the restoration of hand grasp and release in individuals with traumatic tetraplegia has been under development in several laboratories for nearly two decades. ${ }^{2,3}$ Devices that provide restored movement through FES have been termed neuroprostheses. Restoration of hand grasp through

Correspondence: PH Gorman, VA Maryland Health Care System, 10 North Greene Street, Baltimore, Maryland 21201, USA neuroprosthetic technology has been reported to decrease a subject's need for attendant care and adaptive equipment by increasing the number of activities of daily living that subjects can perform independently. 4,5

An ongoing multi center clinical trial to test the use of the neuroprosthesis has established well defined selection criteria for appropriate use of these devices. The estimation of the size of the potential end user population becomes important to both clinicians and manufacturers planning for eventual device distribution. No user population estimates for upper extremity FES system protocols are currently available, although an estimate of the user population for an FES standing system in paraplegia has been published previously by Jaeger et al. ${ }^{6}$

The Rehabilitation Engineering Center at MetroHealth Medical Center (MHMC) and the Department of Veterans Affairs Medical Center in Cleveland, Ohio have been developing and testing upper extremity neuroprostheses for over two decades. In the process of recruiting for research volunteers, every cervical spine patient admitted for rehabilitation at $\mathrm{MHMC}$ was routinely screened for his or her candidacy. 
The purpose of this paper is to review some of the accumulated experiences in the screening process for appropriate FES candidates at a single hospital and thereby arrive at an estimate of the size of the potential end user population for current and future upper extremity FES neuroprostheses. This report examines this screening process over a four and one half year period.

\section{Methods}

The admission database records for the spinal cord injury rehabilitation service at MetroHealth Medical Center were reviewed for the period of January 1987 to June 1991 inclusively. During that period, there were a total of 207 traumatic spinal cord admissions, of which 120 were cervical level injuries. Through review of patient records, interview with the physician director of the spinal cord rehabilitation service, or in many cases interview and examination of the patients themselves, the principal reason for eligibility or exclusion for the upper extremity neuroprosthesis program was determined for all of these subjects. Most of this review was retrospective, although the last year's worth of data was obtained in a prospective manner. All of the retrospectively studied patients had been assessed for their eligibility into the neuroprosthesis program during the time of their initial rehabilitation admission, although a formal screening database had not been kept until the start of this investigation. Any of these early patients for which screening questions were still unanswered were recontacted if possible. The patients seen prospectively were also assessed during their initial rehabilitation stay.

A set of eligibility criteria for inclusion into a neuroprosthesis program had been previously established. ${ }^{7}$ These criteria were initially based on clinical and scientific judgement and have been refined over time with ongoing experience. This selection process includes consideration of anatomic, physiologic, medical, and psychosocial criteria. Also taken into account are the possible alternative methods of restoration of hand function in tetraplegia already available in clinical practice (eg splinting, tendon transfer surgery). Anatomically, patients must have a stable $\mathrm{C} 5$ or $\mathrm{C} 6$ motor level and impairment scale A, B or $\mathrm{C}$ as defined by the Americal Spinal Injury Association (ASIA) and the International Medical Society of Paraplegia (IMSOP). ${ }^{8}$ Although there are some tendon transfer surgeries described in the literature for C5 and C6 tetraplegic individuals, ${ }^{9}$ these procedures have been limited in their success and acceptance. It is this problem that has therefore motivated the development of much of the neuroprosthetic hand grasp technology.

Physiologically, in order for FES to be applied, a sufficient number of forearm and hand muscles must be innervated to allow for electrical stimulation. It has been shown that the stimulation threshold of denervated musculature is considerably higher than that of nerve tissue. ${ }^{10}$ This issue is complex, as the number of innervated muscles necessary to provide adequate grasp depends not only on the innervation distribution but also on the availability of innervated muscles that could be substituted by tendon transfer. ${ }^{11}$ For instance, an innervated but paralyzed flexor carpi ulnaris (FCU) could be used to provide finger flexion by performing tendon transfer surgery and then stimulating the transferred muscle in its new position. Another example would be stimulation of an innervated extensor carpi ulnaris (ECU) in a transferred position to provide finger extension in a subject with a denervated extensor digitorum communis (EDC).

A second physiological issue is the presence of adequate range of motion in joints of the shoulder, arm, forearm, wrist and hand. Some contractures are remediable surgically, such as elbow flexion or forearm supination contractures. Others, especially those which are longstanding and multiple are not surgically manageable and would therefore exclude some candidates.

Medically, patients must be free of overwhelming medical problems such as urosepsis or decubiti that would prevent prolonged wheelchair sitting. They must also possess adequate vision so that they have feedback as to what their hand is doing when it is electrically stimulated since most of these patients lack tactile or proprioceptive sense in the fingers and thumb. Psychosocially, patients who participate in a neuroprosthesis program must be sufficiently motivated to use their hand in daily activities. They must also have adequate family or care giver support to allow for donning of the neuroprosthetic device as part of their daily routine.

The following items were assessed by chart review or examination: age, sex, type of injury, spinal level of injury using both the ASIA/IMSOP classification and the International Classification scheme (see below), ${ }^{12}$ ASIA impairment scale of completeness, and if incomplete, neurologic pattern of injury (ie ASIA clinical syndromes, for example central cord syndrome), innervation status as determined by surface electrical stimulation, medical stability, presence or absence of adequate range of motion, and psychosocial issues such as cognitive ability, extent of family support, motivation level, and travel/logistical considerations.

The International Classification of Hand Function in Tetraplegia was used along with the ASIA/IMSOP classification in this review because it allows for a more detailed description of muscle function in the arm than does the more traditional ASIA/IMSOP motor level scale. The classification scheme is summarized in Table 1.

If during the screening process patients were excluded from eligibility based on an obvious criteria (eg death during rehabilitation hospitalization or improper level of injury), then further screening of 
Table 1 International classification for surgery of the hand in tetraplegia*

\begin{tabular}{lcc}
\hline Group & Muscles & Function \\
\hline 0 & $\begin{array}{c}\text { No muscle below elbow } \\
\text { suitable for transfer }\end{array}$ \\
& Brachioradialis & Elbow flexion \\
1 & ECRL & Wrist extension \\
2 & ECRB & Wrist extension \\
3 & Pronator Teres & Forearm pronation \\
4 & Flexor Carpi Radialis & Wrist flexion \\
5 & EDC & Finger extensors \\
6 & EPL & Thumb extensor \\
7 & FDS or FDP & Partial digital flexors \\
8 & Lacks only intrinsics & \\
9 & Exceptions & \\
$\mathrm{X}$ &
\end{tabular}

*Besides the muscle groups listed, also included in this classification is an indication of the presence of either ocular or cutaneous sensory feedback in the hand. Adapted from ${ }^{12}$

innervation patterns or other criteria was not done. Therefore only a subset of patients received complete screening evaluation.

A battery powered surface electrical stimulator (Med Labs, Inc. Model EMS-2A) was used to evaluate the innervation status of various muscles of the forearm and hand in subjects who met the basic clinical criteria. Since implementation of the neuroprosthesis presently involves hand grasp in only one limb, the reported innervation status represents screening of the stronger limb.

Not all muscles of the forearm and hand on one side have to be electrically excitable for eligibility into the program. Subjects who had some denervation present (ie had some non-stimulatable muscles) might still be candidates for the neuroprosthesis if implementation was performed in conjunction with tendon transfer surgery, as discussed previously. In determining the number of eligible candidates, this factor was considered.

\section{Results}

During the period January 1987 to June 1991, there were a total of 207 initial traumatic spinal cord admissions to the spinal cord injury rehabilitation service, of which 120 were cervical level injuries.

The mean age of these 120 cervical spinal cord injured individuals was $38.6 \pm 18.2$ (SD). Seventy-nine percent of these individuals were male. The etiologies of the injuries for this sample group break down into the following distribution: $51(42.5 \%)$ motor vehicle accident, $26(21.6 \%)$ falls, $14(11.7 \%)$ sport related injuries (including diving accidents), nine (7.5\%) gun shot wounds, ten $(8.3 \%)$ violence by other means, three $(2.5 \%)$ medical or iatrogenic injuries, two $(1.6 \%)$ pedestrian accidents, two (1.6\%) motorcycle accidents, and three $(2.5 \%)$ other causes.
Twenty-seven individuals $(22.5 \%)$ were classified as having a central cord syndrome, and two $(1.7 \%)$ were classified as having a Brown-Sequard syndrome. Of the remaining 91 patients, $49(53.8 \%)$ were considered to have complete (grade A per ASIA Impairment scale) injuries. Of the remaining group, $11(12.1 \%)$ were classified as sensory incomplete only (ie grade B), eight $(8.8 \%)$ were grade $\mathrm{C}$ and $23(25.3 \%)$ were grade D.

Individual selection criteria are listed in Table 2. These criteria were considered for the most part consecutively. For instance, subjects with International classification 5 and above (C7 or lower injury) were eliminated from consideration without further evaluation. For these subjects either other treatments (eg tendon transfer surgery) were available to them or their hand function was sufficient for performance of their activities of daily living that they did not choose to have any surgical intervention.

Of the patients screened, 14 were eventually considered candidates for inclusion into the neuroprosthesis program. Of these, five patients eventually agreed to participate. Table 2 details the reason for elimination from candidacy for all excluded subjects. In addition, the number of ASIA impairment scale D subjects in each exclusionary group is provided (numbers in parentheses).

Initial exclusion criteria, specifically patient death (five) and well recognized incomplete spinal cord injury syndromes (ie central cord and Brown-Sequard - 29), excluded $34(28.4 \%)$ of the study population (cervical injuries). Because current FES technology for

Table 2 User population determination for hand grasp FES: Elimination Table

\begin{tabular}{lccc}
\hline Criteria & $\begin{array}{c}\text { Number } \\
\text { eliminated }^{*}\end{array}$ & $\begin{array}{c}\text { Number } \\
\text { remaining }\end{array}$ & $\begin{array}{c}\text { Percent } \\
\text { remaining }\end{array}$ \\
\hline Tetraplegic & 0 & 120 & 100.0 \\
Patient death & $5(2)$ & 115 & 95.8 \\
Central cord/Brown & $29(29)$ & 86 & 71.7 \\
$\quad$ sequard & $12(2)$ & 74 & 61.7 \\
C2 thru C4 lesion & $27(9)$ & 47 & 39.2 \\
C6 with International & & & \\
$\quad$ Classification $\geqslant 4$ & $15(7)$ & 32 & 26.7 \\
C7 or C8 lesion & $2(2)$ & 30 & 25.0 \\
ASIA Impairment D & & & \\
$\quad$ alone & 4 & 26 & 21.7 \\
Denervation & 2 & 24 & 20.0 \\
ROM problems & 1 & 23 & 19.2 \\
Medical problems & 7 & 16 & 13.3 \\
Psychosocial issues & 2 & 14 & 11.7 \\
Elected tendon transfer & 2 &
\end{tabular}

Total number excluded $=106$ out of 120 patients screened. Since screening for candidacy was done sequentially, if patients were excluded for an initial reason, investigation of eligibility based on other criteria was not necessarily completed. *Numbers in parentheses represent the number of patients within each group that were ASIA impairment level D 
the restoration of hand grasp has been applied at this center only to C5 and some C6 injuries (ie patient with International Classification of 0,1 or 2 ), an additional $53(44.2 \%)$ were excluded for having either functionally too high (12) or too low (42) a level of injury. Of these patients with either too high or too low a lesion, 18 out of $54(33.3 \%)$ had an ASIA impairment scale of $\mathrm{D}$ as well. Two additional patients who were injured at the appropriate level, were nonetheless excluded solely on the basis of ASIA impairment scale (ie group D). Additionally 16 (13.3\%) were excluded for the following reasons: denervated musculature four $(3.3 \%)$, range-of-motion problems two $(1.7 \%)$, over-riding medical problems one $(0.8 \%)$, psychosocial issues seven $(5.8 \%)$, or that the patients chose tendon transfer surgery as an alternative approach to restoring hand grasp two (1.7\%). Psychosocial issues excluding participation included substance abuse, concurrent head injury, or in one instance a patient who left the rehabilitation service against medical advice. Using screening criteria listed above, that left $14(11.7 \%)$ of the cervical SCI patients that were considered candidates for the neuroprosthesis.

\section{Discussion}

Well-defined patient selection criteria and end-user population estimates for upper extremity neuroprostheses using FES technology are becoming more important to physicians and manufacturers as FES devices make the transition from the research laboratory to multi-center clinical trials. This study of 120 tetraplegic patients representing all cervical spinal cord injury patients admitted during 1987 through June 1991 to MetroHealth Medical Center provides a picture of patient characteristics and screening criteria, as well as an estimate of the size of the potential upper extremity neuroprosthesis user population.

The patient population admitted to this SCI rehabilitation unit is matched demographically (ie age, sex, type of injury, injury level, and completeness) to commonly accepted national distributions for spinal cord injury patients. ${ }^{13}$ Thus an estimate of the potential number of users for the current version and application of upper extremity FES can be made. Based on a 1990 estimate for SCI prevalence of 721 cases per million United States population, ${ }^{14}$ of which $55 \%$ are tetraplegics, and a United States population estimate of 263 million, ${ }^{15}$ there are approximately 104,000 tetraplegic individuals in the United States. Given that $11.7 \%$ of the tetraplegics screened in this study were eligible for the neuroprosthesis, approximately 12,200 tetraplegics in America would be eligible for current versions of the neuroprosthetic hand grasp system.

In interpreting these data, several points must be considered. The technology of the neuroprosthetic system used in earlier research protocols is different to that which is being used in clinical trials today. Earlier versions used percutaneous intramuscular electrodes to provide stimulation. ${ }^{16}$ This method was less reliable over the long term because of problems with electrode failure and the electrodes were susceptible to skin site infections. Therefore earlier versions in use during the 1987 to 1991 over which the reported data was collected might have been rejected by patients for reasons that are no longer applicable to current versions of implantable FES. Furthermore, greater experience and acceptance of this type of intervention in tetraplegic individuals over the intervening time also must be considered. Additionally, since the implementation of this hand grasp neuroprosthesis was being carried out under a research protocol, patient decisions regarding whether or not to participate are still influenced by considerations such as the time commitment necessary to participate in a research study that requires extensive additional patient time commitment for data collection purposes. As with any new medical technology, especially when it involves an invasive procedure, justifiable initial patient reluctance lessens as successful clinical experience increases.

The International Classification for Surgery of the Hand in Tetraplegia provides far more useful information for assessing neuroprosthesis eligibility than does the ASIA/IMSOP classification. This is especially true for C6 tetraplegic individuals, who may or may not have active voluntary pronator teres or flexor carpi radialis musculature. Patients with voluntary control of these muscles might be better suited to restorative treatment through tendon transfer surgery alone rather than through the use of a neuroprosthesis. This differentiation would not be possible solely based on the ASIA/IMSOP classification alone. Clearly a careful upper extremity skeletal and neurologic examination is necessary for proper patient selection.

The data presented here is not complete enough to estimate how many individuals would be excluded from neuroprosthesis eligibility on the basis of two or more exclusion criteria. In many cases, once individuals were disqualified for one reason, further evaluation was not performed. Therefore, no definitive conclusion can be drawn (for example) as to how many tetraplegics are sufficiently denervated to exclude them from candidacy on that basis alone. Nonetheless, of the 30 patients remaining after exclusion of patients for reason of death, and inappropriate level or extent of completeness of injury, only four $(13.3 \%)$ were excluded due to excessive denervation. This relatively small proportion is consistent with previous screening information available on lower motor neuron lesions in the arms of tetraplegic individuals. ${ }^{17}$

This study did not address the time interval between spinal paralysis and the screening for a neuroprosthetic system. All of these patients were screened during their initial rehabilitation hospitalization. Actual implementation of implantable neuroprosthetic systems has generally not occurred until at least one year after injury, although the previous percutaneous system was implemented earlier. This 
delay in implementation is consistent with the accepted practice in tetraplegic hand surgery of waiting for the plateau of natural functional recovery to occur prior to surgical intervention. ${ }^{9}$ Nonetheless, screening for use of this type of technology need not wait. Indeed, it is our experience that most (although not all) patients are more receptive to discussion about neuroprostheses early after their injury, before they develop other accommodations to their disability.

These numbers provide some insight into the enduser population for upper extremity neuroprostheses. The numbers are conservative, but do indicate that only a subset of tetraplegic individuals will be candidates for use of this technology. Further development of the neuroprostheses beyond basic hand grasps may provide additional function to SCI candidates at other injury levels and allow application to previously excluded individuals. For example, seven $(5.8 \%)$ of these patients had $\mathrm{C} 4$ level injuries and might benefit from stimulation of muscles such as the deltoid and biceps brachii. In the case of $\mathrm{C} 4$ injuries, extrapolation of the study data would suggest that after screening out for ASIA impairment scale D lesions $(25 \%)$ and other criteria as discussed previously $(10 \%)$ about $32 \%$ of C4 patients representing $3.5 \%$ of the cervical SCI population could be additional viable neuroprosthesis candidates. Extending the indication to individuals with the pronator teres muscle under voluntary control (ie, International Classification motor 4) which is an active direction of current research, would add additional subjects to the pool. Further work with motor incomplete (either central cord syndrome or ASIA impairment scale D) patients might also be a future direction for neuroprosthetic application. Finally, for those patients who are appropriate candidates, the actual participation rate might be greater in the future as the technology becomes more user friendly and more widely accepted, and as the transition occurs from research protocol to clinical implementation.

\section{Acknowledgements}

Special thanks to Dr Frederick Frost, Director of the Spinal Cord Injury Service at MetroHealth Medical Center for his support. Drs Gorman and Peckham and Ms Stroh Wuolle were supported in part by NIDRR grant No H133E 80020 and by the Department of Veterans Affairs Rehabilitation Research and Development Service. Dr Gorman was additionally supported by a NIDRR Mary E Switzer Rehabilitation Research Fellowship Award during this work.

\section{References}

1 Hansen RW, Franklin MR. Sexual loss in relation to other functional losses for spinal cord injured males. Arch Phys Med Rehab 1976; 57: 291 - 293 .

2 Keith MW et al. Functional Neuromuscular Stimulation Neuroprosthesis for the Tetraplegic Hand. Clin Ortho Related Research 1988; 233: $25-33$.

3 Gorman PH, Peckham PH. Upper Extremity Functional Neuromuscular Stimulation. J Neuro Rehab 1991; 5(1\&2): 3-11.

4 Wijman CA et al. Functional evaluation of quadriplegic patients using a hand neuroprosthesis. Arch Phys Med Rehab 1990; 71: $1053-1057$.

5 Mulcahey MJ et al. Functional Neuromuscular Stimulation: Outcomes in Young People with Tetraplegia. J Am Paraplegia Soc 1994; 17 20-35.

6 Jaeger RJ, Yarkony GM, Roth EJ, Lovell L. Estimating the User Population of a Simple Electrical Stimulation System for Standing. Paraplegia 1990; 28: $505-511$.

7 Stroh KW et al. Candidate Selection for Use with a Functional Neuromuscular Stimulation Hand System. Proceedings ICAART 1991; Montreal: pp 330-331.

8 Ditunno JF, Young W, Donovan WH, Creasey G. The International Standards Booklet for Neurological and Functional Classification of Spinal Cord Injury. Paraplegia 1994; 32: $70-80$.

9 Freehafer AA, Peckham PH, Keith MW. New Concepts on Treatment of the Upper Limb in the Tetraplegic: Surgical Restoration and Functional Neuromuscular Stimulation. Hand Clinics 1988; 4(4): 563-574.

10 Crago PE, Peckham PH, Mortimer JT, Van Der Meulen JP. The choice of pulse duration for chronic electrical stimulation via surface, nerve and intramuscular electrodes. Annals Biomed Engineering 1974; 3: 252 - 264.

11 Keith MW et al. Tendon Transfers and Functional Electrical Stimulation for Restoration of Hand Function in Spinal Cord Injury. J Hand Surg 1996; 21A 89-99.

12 McDowell CL, Moberg EA, House JH. The Second International Conference on surgical rehabilitation of the upper limb in tetraplegia (quadriplegia). J Hand Surg 1985; 11A 604-608.

13 Stover SL, Fine PR. Spinal Cord Injury: Facts and Figures. The University of Alabama at Birmingham: Birmingham, Alabama 1986.

14 Harvey C, et al. New Estimates of Traumatic SCI Prevalence: A Survey Based Approach. Paraplegia 1990; 28: 537-544.

15 July 1995 estimate, from U.S. Bureau of the Census, Current Population Reports, pp. 25-1104, Population Projection of the United States, by age, sex, race, and Hispanic Origin: 1993 to 2050, US Government Printing Office, Washington, DC, 1993.

16 Memberg WD et al. An Analysis of the Reliability of Percutaneous Intramuscular Electrodes in Upper Extremity FNS Applications. IEEE Trans Rehab Engineering 1993; 1(2): $126-132$.

17 Peckham PH, Mortimer JT, Marsolais EB. Upper and Lower Motor Neuron Lesions in the Upper Extremity Muscles of Tetraplegics. Paraplegia 1976; 14: 115-121. 